\title{
Implementation of Convolutional Neural Network to Classification Gender based on Fingerprint
}

\author{
Ahmad Ilham Gustisyaf \\ Widyatama University, Bandung, 40125, Indonesia \\ Email: ilham.gustisyaf@widyatama.ac.id \\ Ardiles Sinaga \\ Widyatama University, Bandung, 40125, Indonesia \\ Email: sinaga.diles@gmail.com
}

Received: 11 April 2020; Accepted: 25 June 2020; Published: 08 August 2021

\begin{abstract}
Gender is one of the vital information to identify someone. If we can decide with conviction whether an individual is male or female, it will restrain the inquiry list and abbreviate the pursuit time. The way toward distinguishing fingerprints is one of the significant, simple to do assortment strategies, the cost is cheap, and a dactyloscopy authority does the particular outcome. The classification of the image gets the issues in computer vision, where a computer can mimic the capacity of an individual to comprehend the data in the image. Process of classifying image can be performing with deep learning where the process like the working of the brain in thinking and trying to reproduce part of its functions by using units associated with relationship, like a neuron. Convolutional neural network is one type of deep learning. In this research, will be doing to classification gender based on fingerprint using method Convolutional Neural Network, and then we will make three models to determined gender, with a total of 49270 image data that included test data and training data by classifying two categories, male and female. Of the three models, we are taking the highest accuracy to use in making this application. Results of this research is we get Model2 will be used as a model CNN with the accuracy level of $99.9667 \%$.
\end{abstract}

Index Terms: Convolutional Neural Network, Classification Gender, Fingerprint, Deep Learning, TensorFlow.

\section{Introduction}

Gender is one of the vital information to identify someone. If we can decide with conviction whether an individual is male or female, it will restrain the inquiry list and abbreviate the pursuit time while improving the probability of someone is being recognized[1].

To identify someone, we can do it with biometrics. Biometric is physical or conduct human attributes that can utilize to carefully recognize an individual to allow access to system, device, or data[2]. Instances of these biometric identifiers are fingerprints, facial patterns, voice, or typing cadence. Every one of these identifiers is view as attractive to the individual, and they might be utilized in the blend to guarantee more noteworthy exactness od recognizable proof.

In this research, we did it utilizing fingerprint images because the way toward distinguishing fingerprints is one of the noteworthy, simple to do assortment strategies, the cost is cheap, and a dactyloscopy authority does the particular outcome. On the off chance that a basic unique mark check as done right now be utilized by specialists toward the start of the assessment to decide the gender of the injured individual, it will significantly help the assessment procedure. Additionally, fingerprint, even though their shape and size of the fingerprint may get change, however, which doesn't influence the ridge pattern[3].

In general, the classification of the image gets one of the issues in computer vision, where a computer can mimic the capacity of an individual to comprehend the data in a picture, for example, cat, dog, tree, vehicle, or recognize specific patterns in the image. For people, this turns into a basic and straightforward activity. However, for the computer, this is a troublesome activity for a computer because a computer can observe pixel esteems and pixel information in a picture, so it is hard to process[4].

This image classification process can be doing with Discrete Wavelet Transform (DWT)[5], Support Vector Machine (SVM)[6], manifold learning method - Locally Linear Embedding (LLE)[7], Deep Learning[8]. In this research, deep learning will be using because deep learning goes through a robust computational process, has a more 
profound model training process, and in recent years it is often used to create models for the problem of classifying images[9]. Deep learning is like the working of the brain in thinking and trying to reproduce part of its function by using units associated with relationships, like a neuron[10-12]. Convolutional neural network is one type of deep learning[13].

Research on the classification of fingerprint object image has been carrying out several times, including by WangSu Jeon and Sang-Yong Rhee in 2017. This research discusses classifying fingerprint patterns using Convolutional Neural Network method. In this paper, they are proposing the use of a convolutional neural network model combined with an ensemble model and a batch normalization technique after minimizing the number of quality improvement processes needed for fingerprint images, which operate more closely with human perception. The result is that three models were creating through modification was applied using each model. In this research, they found that fingerprint preprocessing is very necessary after comparing models one and two. Model three has a small but fast batch size and shows excellent performance. By using model three, the average classification level is 97,2\%[14].

Based on the problems that have been explaining above, the formulation of the problem are how to determine the best model to be used in detecting fingerprint images to classification the gender of someone using convolutional neural network, and how the accuracy of detecting fingerprint images to determine the gender of someone using convolutional neural network.

The major research objectives are to be able to find out the best model to be used in detecting fingerprint images to classification the gender of someone using convolutional neural network and to able to know the level of accuracy of detecting fingerprint images to classification gender someone using convolutional neural network.

The main limitation of the research is using data in the form of fingerprint photo samples, this application only uses a bitmap image of type *.BMP, the data collection method used in this research from the 'Kaggle' web, and using TensorFlow as a tool.

Therefore, in this research, we will making three models to determined gender based on fingerprint using method convolutional neural network, with a total of 49270 image data that included test data and training data by classifying two categories, male and female[15]. Of the three models, we are taking the highest accuracy to use in making this application.

\section{Literature Review}

Research on the classification of an object image had previously been conducting by Laila Marifatul Azizah, Sitti Fadillah Umayah, and Febriyana Fajar in 2018. This research uses CNN (Convolutional Neural Network) as a method of deep learning to detect the surface of mangos teen peel. Our research was founding a configuration that is the best accuracy value. This technique accomplished the most extreme incentive by parameter 4 layers and epoch value of 30 . From this experiment, the test results for skin detection are $98 \%$ accuracy. Suggestions from this research for the next that is: testing with other parameters in the CNN classification, which is then combined with the most appropriate feature extraction for use in the classification process to produce optimal accuracy. The addition of epoch for more optimal results. Maximize the amount of data up to 1000 image data with high-quality photo quality[16].

Then the research was conducted by Wang-Su Jeon and Sang-Yong Rhee in 2017. This research discusses classifying fingerprint patterns using the convolutional neural network. In this paper, they propose the use of a convolutional neural network model combined with an ensemble model and a batch normalization technique after minimizing the number of quality improvement processes. The result is that three models were creating through modification was applied using each model. In this research, they found model three has a small but fast batch size and shows excellent performance. They using model three, the average classification level is $97,2 \%$. Suggestion from this research for the next that is: study CNN structure optimization, quicken learning speed for quick coordinating, and examine strategies to improve image the classification execution with noise[14].

Research on the classifying gender based on fingerprint has previously been conducting by Ms. Bindhu K. Rajan, Ms. Nimpha Anto, dan Ms. Sneha Jose in 2014. This research discusses determining gender based on fingerprints and irises using the Ridge Thickness to Valley Thickness Ratio (RTVTR). The result of this research is finding the best vector features to use on this problem. Suggestion from this research for the next that is: to be able to improve the system by investigating better feature and improving classification[17].

\section{A. Biometric}

A Biometric system is a self-recognition technology using body parts or human behavior. Fingerprints and signatures, each of which is an example of biometrics-based on body parts and human behavior. Biometric comes from the word bio and metrics. Bio means something alive, and metrics means to measure. Biometrics means measuring the distinguishing characteristics (distinguish traits) on a person's body or behavior that is using to automatically recognize the person's identity, by comparing it with characteristics that have been previously storing in a database.

The fingerprint is a characteristic commonly used in biometrics systems. The introduction of fingerprint characteristics has been using since the early $19^{\text {th }}$ century, which is one of the oldest biometrics systems and has been using throughout the world, which has proven to be very useful in individual recognition. At present, research on 
biometric systems using fingerprints has increased significantly, this matter because the fingerprint is unique characteristics, more stable, and more accurate than other characteristics[18].

\section{B. Fingerprint}

The fingerprint is one of human identify which cannot be changed. "No human in this world has the same fingerprint". This expression reveals that every human being has a different fingerprint. The fingerprint is the specialty of every human being. According to Reinhard Hutagaol, the fingerprint is skin thickened and thinned to form a ridge on the palm of a fingerprint forming a pattern. Fingerprint will not disappear until a person dies and rot scratches or usually cuts when the skin changes will form the same pattern. However, fingerprint can be damaged because the skin severely burns[19]. There are two layers of fingerprint skin, the dermal layer and the epidermal layer[20].

The dermal layer is hides skin (actual skin). This skin determines the lines that exist on the surface of the palm skin. Then the epidermal layer is the outer skin layer (papillary line). The papillary line is a line that becomes a person's daily life to determine the first formulation and comparison of fingerprints. The fingerprints have three kinds, namely visible impression, latent impression, and plastic impression[20].

The visible impression is a fingerprint that can be seen directly without using tools. Whereas, the latent impression is a fingerprint that usually cannot be seen immediately but must use several methods of development in advance so that it can appear more clearly. And then the plastic impression is fingerprint imprinted on soft objects such as soap, candy, and other objects.

\section{Deep Learning}

Deep learning is one of the Artificial Intelligence (AI) work that mimics the activities of the human mind in preparing information and making designs for use in dynamic. Deep learning is a subset of Machine Learning in artificial intelligence that has systems fit for taking in solo from information that is unstructured or unlabeled data[21].

Deep learning has become a challenge to define the meaning of deep learning for many people because its understanding has changed slowly over the past decade. With the definition of deep learning, which states that: neural networks with more two layers; making deep learning sound has been around since the 1980s. Neural networks have a better side in terms of architecture that the previous network (more in processing power); it was proving in recent years that the result is optimal more and it's also suitable for problem solving with image recognition, especially on a large scale[22, 23].

\section{TensorFlow}

TensorFlow is a software library developed by the Google Brain Team in the Google Intelligent Engine research organization to carry out machine learning and deep neural network research[24]. TensorFlow combines computational algebra optimization techniques to compile, making it easier to calculate many mathematical expressions where the problem is the time needed to do the calculation. The main features of TensorFlow are as follows[25]:

1. Define, optimize, and calculate efficiently mathematical expressions involving multi-dimension arrays (tensors).

2. Programming supporting deep neural networks and machine learning techniques.

3. Using a transparent Graphics Processing Unit (GPU) automates the management and optimization of the same memory and data used. TensorFlow can write the same code and run it on either the Central Processing Unit (CPU) or GPU. More specifically, TensorFlow will know which part of the calculation must be moving to the GPU.

4. High computing scalability across machines and large data sets.

\section{E. Research Method}

The research method is used to be able to help the problem so that the result obtained is more systematic and directed, the following Fig. 1 is a research method process. 


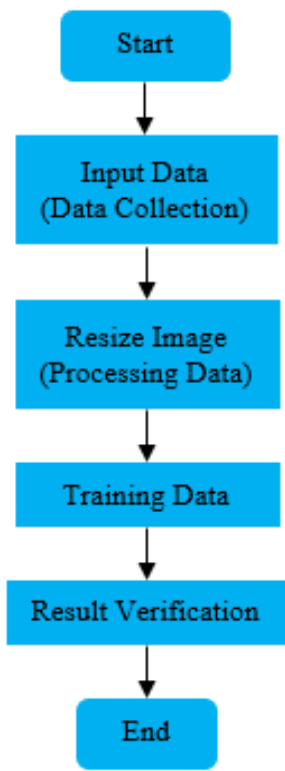

Fig. 1. Research Method

\section{Data Collection}

In conducting research, the first step is collecting data in the form of a set of fingerprint image.

\section{Preprocessing Data}

Before entering the training data, preprocessing data is performed, which is resize the fingerprint image $96 \times 96$ pixels.

\section{Training Data}

In this processing the dataset will be trained using the convolutional neural network methodology. This training data processing is a stage where convolutional neural network is trained to obtained high accuracy from the classification conducted.

\section{Result Verification}

After doing all the above process, at this stage, we will display the results of gender prediction based on fingerprint images in the application that has been making.

\section{Analysis}

\section{A. Convolutional Neural Network}

Convolutional Neural Network is including in the type of deep learning because of the depth of the network[26]. Convolutional neural network is a convolution operation that combines several layers of processing, using several elements that operate in parallel and are inspired by the biological nervous system[27]. On convolution neural network each neuron is presented in two-dimensional form, so this method is suitable for processing with input in the form of images[28].

Convolutional neural network has three layers, that is convolution layer, pooling layer, and fully connected layer. Generally, convolutional neural network process flow in Fig. 2 and can also be formulated calculated in equation (1):

$$
\mathrm{S}(i, j)=(I * K)(i, j)=\sum_{m} \sum_{n} I(m, n) K(i-m, j-n)
$$

$\mathrm{I}=$ image input

$\mathrm{K}=$ Kernel or filter used

$\mathrm{m}=$ line in the image

$\mathrm{n}=$ column in the image 


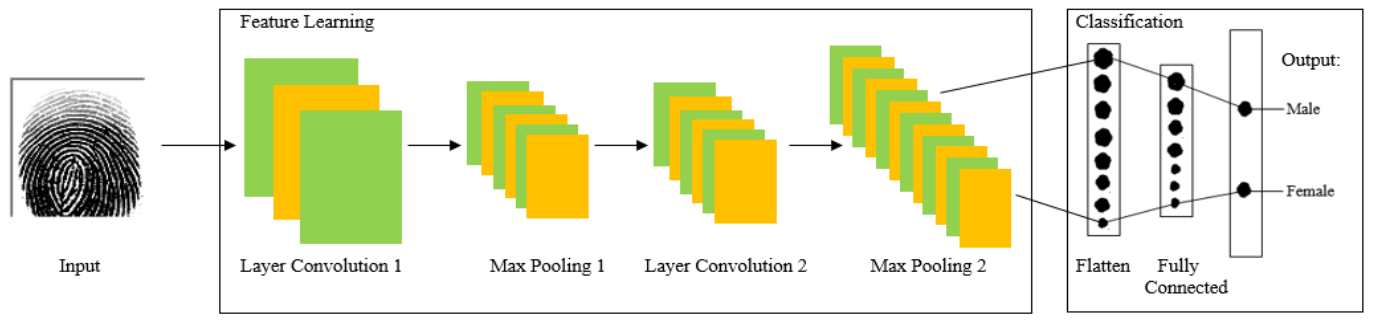

Fig. 2. Convolutional Neural Network

Hyperparameter is a parameter whose worth is resolving before the start of the learning[29]. Usually, the hyperparameter is circulating outside the model. Hyperparameter drastically influences the speed and nature of the procedure of deep learning[13]. In this research, we use Adam as an optimizer with a learning rate of 0.00001. In this research, using Binary Cross-Entropy Loss are in equation (2) and equation (3):

$$
\begin{gathered}
C E=-\sum_{i=1}^{c^{\prime}=2}-t_{1} \log \left(f\left(s_{1}\right)\right)-\left(1-t_{1}\right) \log \left(1-f\left(s_{1}\right)\right) \\
f(s)_{i}=\frac{1}{1+e^{-s_{i}}}
\end{gathered}
$$

The above equation becomes the connecting channel for each class $\mathrm{C}$. Then set $\mathrm{C}$ independent binary classification issues $\left(C^{\prime}=2\right)$. At the point, summarize the loss over the distinctive binary issues: summarize the gradients of each binary item to backpropagate, and the losses to screen the global loss. $S_{1}$ and $t_{1}$ are the scores, and the ground truth label for the class $\mathrm{c} 1$, which is additionally the class $C_{i}$ in C. $s_{2}=1-s_{1}$ and $t_{2}=1-t_{1}$ is the score and the ground truth name of the class $C_{2}$, which isn't a class in our unique issue with $C$ classes, yet a class make to set up the binary point with $c_{1}=c_{i}$.

Fingerprint classification can evaluate from class distribution and classifier confusion matrix. At that point, the level of penetration of identification is complete. Equation (4) is assessing the average penetration rate of info fingerprint images in the ' $i$ ' class of class ' $m$ '. Equation (5) is the average penetration rate in every possible class.

$$
\begin{gathered}
r_{i}^{-}=1+q_{i}\left(1-p_{i}\right) i \in\{1, \ldots, m\} \\
\tilde{r}=\sum_{i=1}^{m} p i \tilde{r} i
\end{gathered}
$$

$P_{i}$ is the fingerprint extent in class $i$, and the accuracy rate of that class is giving by $q_{i} \cdot q_{i}=1$ and $r_{1}{ }^{-}=p_{i}$ is the best alluring situation which maintains a strategic distance from the misclassification of contributions of class i. this in turn results in the penetration rate for that class in consistently $\mathrm{p}_{\mathrm{i}}$.

\section{Convolution Layer}

Convolution layer is a process of extracting an object from the input image using a filter. This filter contains weights used to detect the character of an object by multiplication between two metrices. The first matrix is an input image, and the second is a filter producing a feature map[10]. Fig. 3 is a process convolution layer.

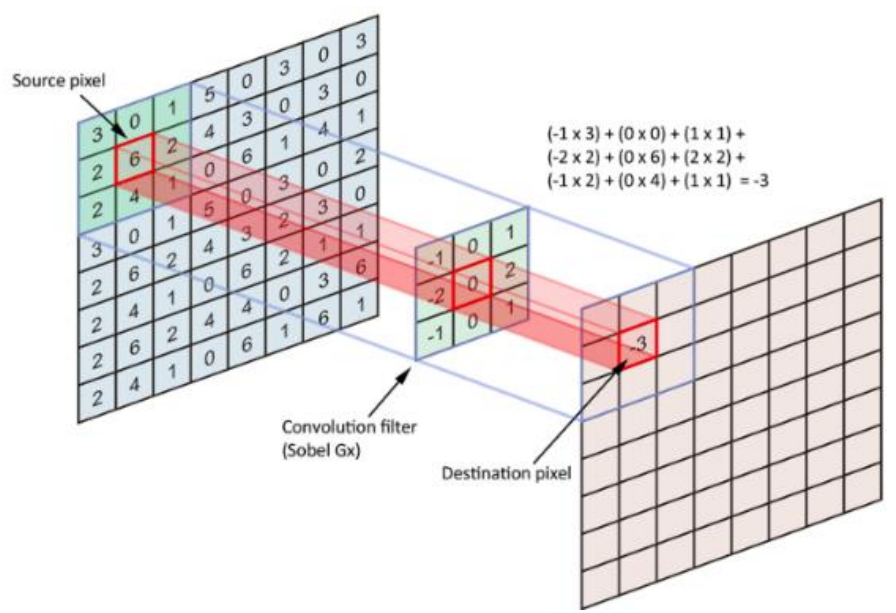

Fig. 3. Convolution Layer, Source: mc.ai/ai-starter-build-your-first-convolution-neural-network-in-keras-from-scratch-to-perform 


\section{Pooling Layer}

At this layer, the size of a data image from the convolution process will be reducing. The pooling layer consists of filters of a specific size and stride then shifts throughout the feature map area. For most Convolutional Neural Network architectures, the pooling method used is max pooling. Max pooling divides the convolution output layer into several gird, and then each filter shift will take the most considerable value from each grid. A small portion of the image size produced int his process is useful for reducing the dimensions of the data so that it can reduce the number of parameters in the next step. The output of the pooling layer is a feature map[4]. Fig. 4 is a process pooling layer.

Image Matrix
\begin{tabular}{|l|l|l|l|}
\hline 2 & 1 & 3 & 1 \\
\hline 1 & 0 & 1 & 4 \\
\hline 0 & 6 & 9 & 5 \\
\hline 7 & 1 & 4 & 1 \\
\hline
\end{tabular}

Max Pool

\begin{tabular}{|l|l|}
\hline 2 & 4 \\
\hline 7 & 9 \\
\hline
\end{tabular}

Fig. 4. Pooling Layer. Source: austingwalters.com/convolutional-neural-networks-cnn-to-classify-sentences

\section{Fully Connected Layer}

The feature map, which is output of pooling layer, is still in the form of a multidimensional array. This layer reshapes the feature map and generates $n$-dimensional vectors where $n$ is the number of output classes the program must select. The result is a feature map in the form of a one-dimensional vector[30]. Fig. 5 is a process fully connected layer.

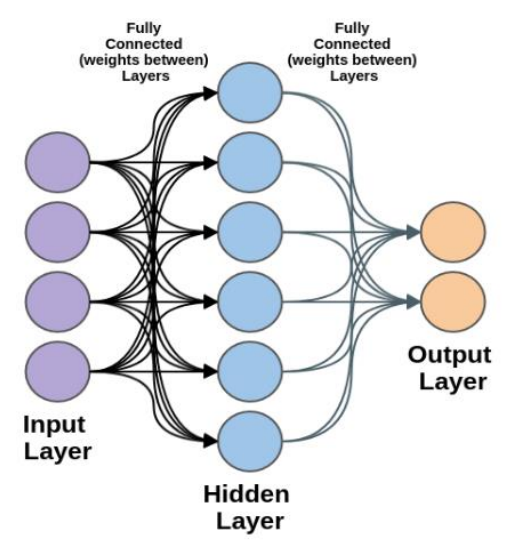

Fig. 5. Fully Connected Layer, Source: austingwalters.com/convolutional-neural-networks-cnn-to-classify-sentences

\section{Performance Evaluation Indexes}

To evaluate the performance of a CNN, we can use the confusion matrix. In confusion matrix, there are several terms True Positive (TP), False Positive (FP), True Negative (TN), and False Negative (FN). TP is something that is predicting to correct, and the result is correct. FP is something that is predicting to correct, but the result is wrong. TN is something that is predicting to wrong, and the result is wrong. FN is something that is predicting to wrong, but the result is correct. With the confusion matrix, we can determine Accuracy, Recall, Precision, and F1 Score[31].

Accuracy is the calculation of the correct prediction ratio with the aggregate data. Recall is the calculation of the ratio of true positive predictions compared to overall positive true data. Precision is the calculation of the ratio of true positive predictions compared to overall positive predicted results. F1 Score is a calculation of the weight average comparison of precision and recall[32]. Equation (6) until equation (9) are calculation of accuracy, recall, precision, and F1 Score.

$$
\begin{gathered}
\text { Accuracy }=\frac{(T P+T N)}{(T P+F P+T N+F N)} \\
\text { Recall }=\frac{T P}{(T P+F N)} \\
\text { Precission }=\frac{T P}{(T P+F P)}
\end{gathered}
$$




$$
F 1 \text { Score }=2 \times \frac{(\text { Precission } \times \text { Recall })}{(\text { Precission }+ \text { Recall })}
$$

\section{B. System Planning}

\section{Data Collection}

In conducting research, the first step is collecting data in the form of a set of fingerprint image. This dataset is useful as an input that will process on the system. The dataset in this research was a source from Kaggle, which is a gender classification competition website based on fingerprints this dataset has a total of 49270 images data that included test data and training data by classifying two categories, male and female. Fig. 6 is an example of a gender male fingerprint image, and Fig. 7 is an example of a gender female fingerprint image.

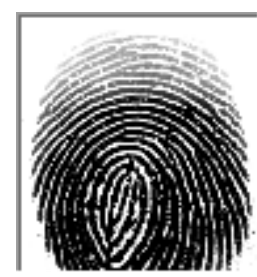

Fig. 6. Gender Male

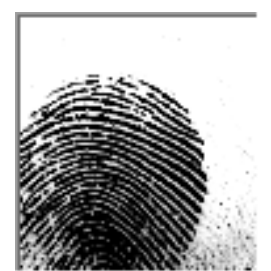

Fig. 7. Gender Female

\section{Preprocessing Data}

The dataset used has an image size of 96x103 pixels. This size image needs to reduce to facilitate the training process. Before entering the training data, preprocessing data is performed, which is resize the fingerprint image $96 \times 96$ pixels.

\section{Training Data}

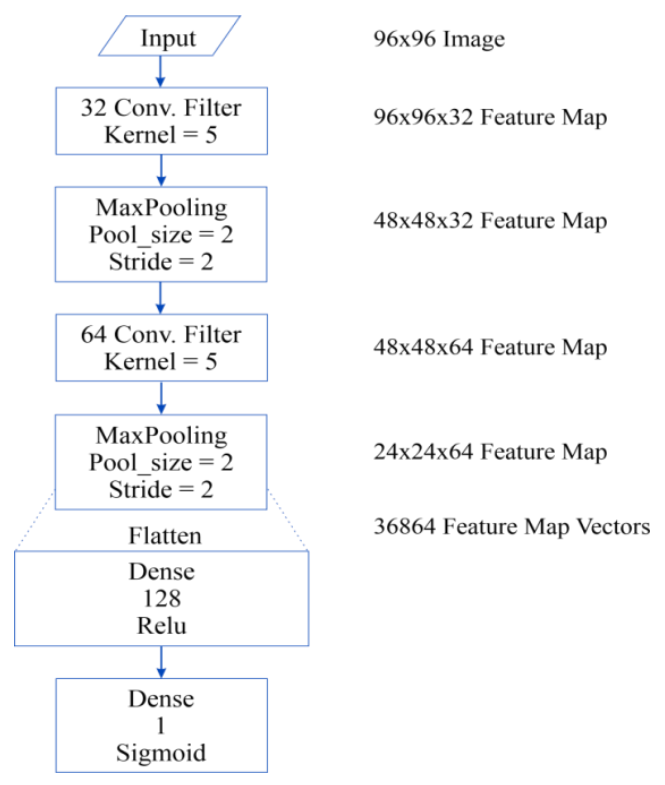

Fig. 8. The Process of Scenario Model1

In this processing the dataset will be trained using the convolutional neural network methodology. This training data processing is a stage where convolutional neural network is trained to obtained high accuracy from the classification conducted. Convolution is the first thing to do in this research. Then the pooling process, which uses the 
max-pooling. And the fully connected layer process, here using, flattens, and doing 2 stage dense, dense with RELU (Ratified Linear Unit) activation, and dense using sigmoid activation. In this research, we will make three models CNN. Fig. 8 is a process of the model scenario used in Model1. Fig. 9 is a process of the model scenario used in Model2. Fig. 10 is a process of the model scenario used in Model3.

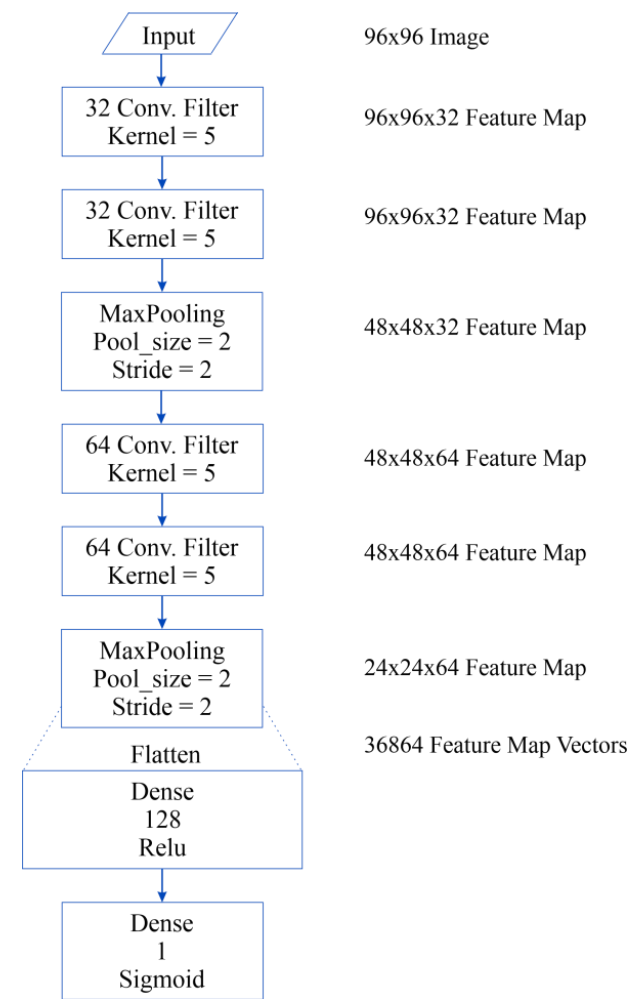

Fig. 9. The Process of Scenario Model2

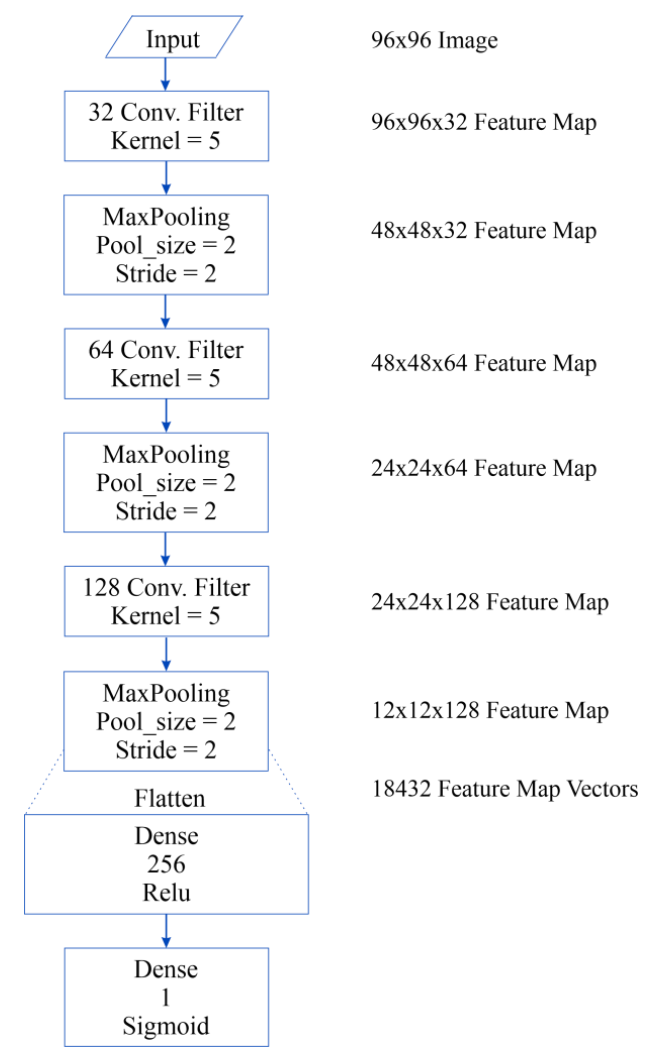

Fig. 10. The Process of Scenario Model3 
Table 1 is the result of the scenario Model1 that has been making.

Table 1. Result of Scenario Model1

\begin{tabular}{ccc}
\hline Layer & Size & Parameter \\
\hline Conv2d_1 & $96,96,32$ & 832 \\
\hline Max_pooling2d_1 & $48,48,32$ & 0 \\
\hline Conv2d_2 & $48,48,64$ & 18496 \\
\hline Max_pooling2d_2 & $24,24,64$ & 0 \\
\hline Flatten_1 & 36864 & 0 \\
\hline Dense_1 & 128 & 4718720 \\
\hline Dense_2 & 1 & 129 \\
\hline Total & & 4738177 \\
\hline
\end{tabular}

Table 2 is the result of the scenario Model 2 that has been making.

Table 2. Model2

\begin{tabular}{ccc}
\hline Layer & Size & Parameter \\
\hline Conv2d_1 & $96,96,32$ & 832 \\
\hline Conv2d_2 & $96,96,32$ & 25632 \\
\hline Max_pooling2d_1 & $48,48,32$ & 0 \\
\hline Conv2d_3 & $48,48,64$ & 18496 \\
\hline Conv2d_4 & $48,48,64$ & 36928 \\
\hline Max_pooling2d_2 & $24,24,64$ & 0 \\
\hline Flatten_1 & 36864 & 0 \\
\hline Dense_1 & 128 & 4718720 \\
\hline Dense_2 & 1 & 129 \\
\hline & & 4800737 \\
\hline
\end{tabular}

Table 3 is the result of the scenario Model 3 that has been making.

Table 3. Model3

\begin{tabular}{ccc}
\hline Layer & Size & parameter \\
\hline Conv2d_1 & $96,96,32$ & 832 \\
\hline Max_pooling2d_1 & $48,48,32$ & 0 \\
\hline Conv2d_2 & $48,48,64$ & 51264 \\
\hline Max_pooling2d_2 & $24,24,64$ & 0 \\
\hline Conv2d_3 & $24,24,128$ & 73856 \\
\hline Max_pooling2d_3 & $12,12,128$ & 0 \\
\hline Flatten_1 & 18432 & 0 \\
\hline Dense_1 & 256 & 4718848 \\
\hline Dense_2 & 1 & 257 \\
\hline Total & & 4845057 \\
\hline
\end{tabular}

\section{Output Prediction}

In this process, to display the result of fingerprint image that have been inputting. Fig. 11 is the first display of the Graphical User Interface (GUI) that made this application. Fig. 12 shows the screen of a GUI this application after the image has been inputting. 


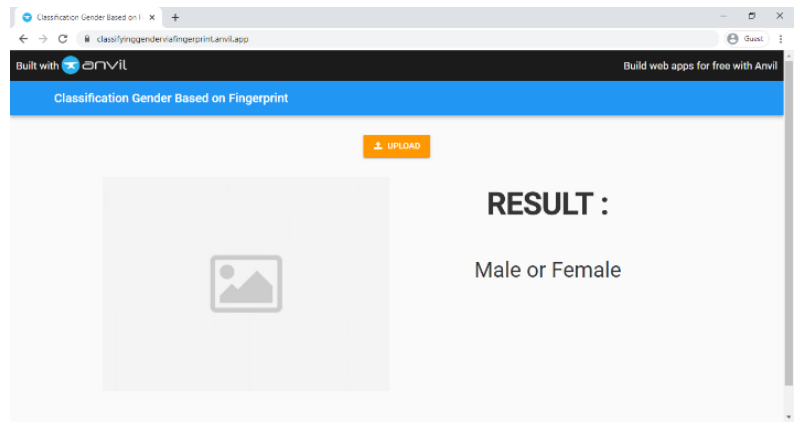

Fig. 11. Graphical User Interface (GUI)

Fig. 12. Graphical User Interface

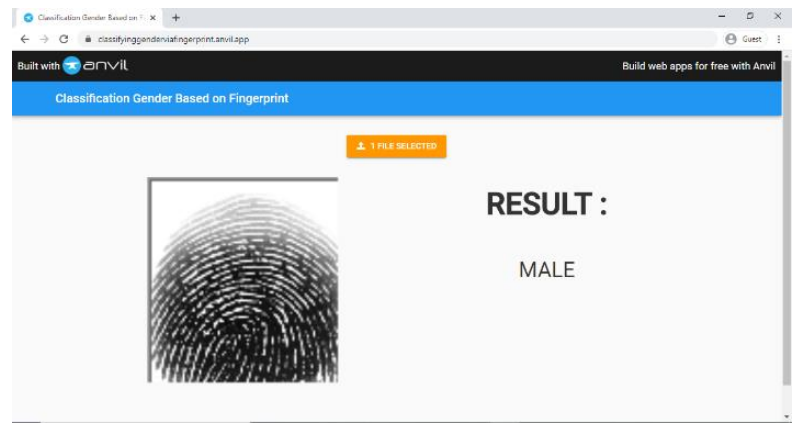

The input button is a shortcut for entering the fingerprint image. Then, the system will do the work according to the order of the methods from 1 to 3 previously described. And do the output by displaying an image that were already inputting and the result of fingerprint image prediction.

\section{Results \& Discussion}

The training process on the convolutional neural network method aims to make convolutional neural network recognize a dataset for the training process so that it can form an optimal training model. To evaluating the accuracy of the convolutional neural network architectures model, a dataset of 49270 fingerprint image data was using. In comparison, the comparison of its use divided into 39416 training data and 9854 validation data.

All the convolutional neural network training model runs 30 epochs using the TensorFlow library. From the test, the accuracy and loss values generating. Fig. 13 is a comparison of the value training accuracy, validation accuracy, training loss, and validation loss of Model1.

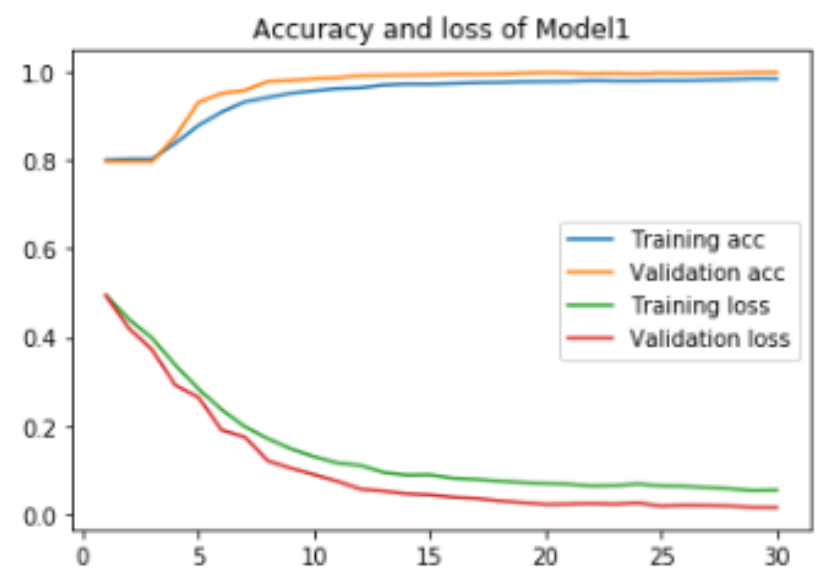

Fig. 13. Comparation of the value accuracy and loss of Model1 
Fig. 14 is a comparison of the value training accuracy, validation accuracy, training loss, and validation loss of Model2.

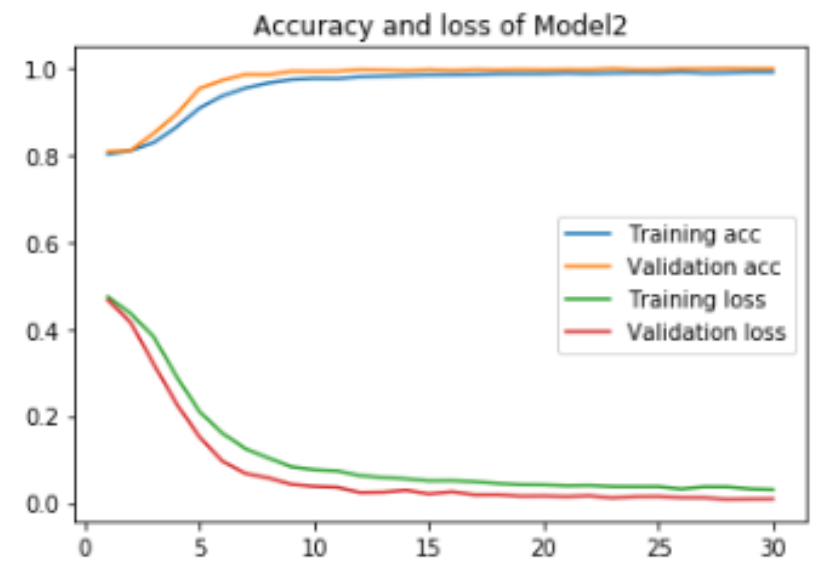

Fig. 14. Comparation of the value accuracy and loss of Model2

Fig. 15 is a comparison of the value training accuracy, validation accuracy, training loss, and validation loss of Model3.

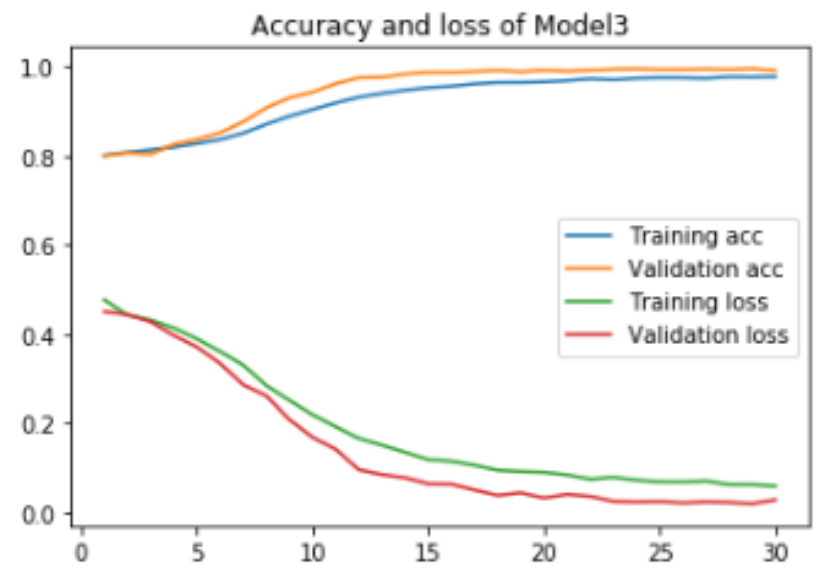

Fig. 15. Comparation of the value accuracy and loss of Model3

Based on the graphic images above, then in table 4 is the result of the final results of each Model CNN that we have worked on this research.

Table 4. Result of Testing on Every Model

\begin{tabular}{ccccc}
\hline $\begin{array}{c}\text { Name of } \\
\text { Model }\end{array}$ & $\begin{array}{c}\text { Training } \\
\text { Acc }\end{array}$ & $\begin{array}{c}\text { Training } \\
\text { Loss }\end{array}$ & $\begin{array}{c}\text { Validation } \\
\text { Acc }\end{array}$ & $\begin{array}{c}\text { Validation } \\
\text { Loss }\end{array}$ \\
\hline Model1 & 0.9682 & 0.0884 & 0.9943 & 0.0259 \\
\hline Model2 & $\mathbf{0 . 9 9 1 1}$ & $\mathbf{0 . 0 2 9 3}$ & $\mathbf{0 . 9 9 6 8}$ & $\mathbf{0 . 0 1 3 0}$ \\
\hline Model3 & 0.9724 & 0.0732 & 0.9942 & 0.0274 \\
\hline
\end{tabular}

Table 5 is the result of accuracy, recall, precision, and F1 Score of each model CNN that we have worked on this research.

Table 5. Result of Accuracy, Recall, Precision, and F1 Score on Every Model

\begin{tabular}{ccccc}
\hline $\begin{array}{c}\text { Name of } \\
\text { Model }\end{array}$ & $\begin{array}{c}\text { Accuracy } \\
(\boldsymbol{\%})\end{array}$ & $\begin{array}{c}\text { Recall } \\
(\boldsymbol{\%})\end{array}$ & $\begin{array}{c}\text { Precision } \\
(\mathbf{\%})\end{array}$ & $\begin{array}{c}\text { F1 Score } \\
(\boldsymbol{\%})\end{array}$ \\
\hline Model1 & 99.7333 & 98.7159 & 100 & 99.3538 \\
\hline Model2 & $\mathbf{9 9 . 9 6 6 7}$ & $\mathbf{9 9 . 8 3 7 7}$ & $\mathbf{1 0 0}$ & $\mathbf{9 9 . 9 1 8 8}$ \\
\hline Model3 & 99.8833 & 99.7559 & 99.6748 & 99.7153 \\
\hline
\end{tabular}


Based on table 4 and table 5, then Model2 has highest accuracy, with 0.9911 training accuracy, 0.0293 training loss, 0.9968 validation accuracy, 0.0130 validation loss, $99.9667 \%$ accuracy, $99.8377 \%$ recall, $100 \%$ precision, and 99.9188\% F1 Score.

Therefore, Model 2 will be using as a CNN model which will use in an application that will be made to classification a gender someone based on fingerprint images and also Model2 has an accuracy of $99.9667 \%$.

\section{Conclusion}

Based on the result of research conducted to classifying gender someone based on a fingerprint, using the convolutional neural network with making three models CNN, we get Model2 to have highest level of accuracy, that is $99.9667 \%$.

Thus, it shows the convolutional neural network training model that was forming with a combination of hyperparameter used (Adam optimizer with learning rate 0.00001 , and the loss function with binary entropy loss) has a high level of evaluation accuracy. It can use as a problem solving in this research, specifically can know the best model to use in detecting fingerprint image to classification gender someone using CNN, and to know the level of accuracy of detecting fingerprint images to classification gender someone using CNN.

Suggestions for further research are as follows: Added the data augmentation stage, namely zoom, and flip to improve accuracy in this research. And this application can only be used for the *.BMP format image, so hopefully, it can be using for another image format.

\section{References}

[1] D. I. Marbun, D. Ilmu, K. Forensik, F. K. Usu, and R. H. A. Malik, "Penentuan Jenis Kelamin Berdasarkan Kerapatan Alur Sidik Jari," Maj. Kedokt. Nusant. J. Med. Sch., vol. 51, no. 1, pp. 6-9, 2019.

[2] D. Scarlet, "What is biometrics? 10 physical and behavior identifiers that can be used for authentication," Journal of Chemical Information and Modeling, 2013. [Online]. Available: https://www.csoonline.com/article/3339565/what-is-biometrics-and-whycollecting-biometric-data-is-risky.html. [Accessed: 29-Feb-2020].

[3] M. K. Shinde and S. A. Annadate, "Analysis of fingerprint image for gender classification or identification: Using wavelet transform and singular value decomposition," Proc. - 1st Int. Conf. Comput. Commun. Control Autom. ICCUBEA 2015, pp. 650-654, 2015, doi: 10.1109/ICCUBEA.2015.133.

[4] A. Santoso and G. Ariyanto, "Implementasi Deep Learning Berbasis Tensorflow," J. Emit., vol. 18, no. 01, pp. $22-27,2018$.

[5] X. Cao, L. Xu, D. Meng, Q. Zhao, and Z. Xu, "Integration of 3-dimensional discrete wavelet transform and Markov random field for hyperspectral image classification," Neurocomputing, vol. 226, no. July, pp. 90-100, 2017, doi: 10.1016/j.neucom.2016.11.034.

[6] Z. Li, B. Niu, F. Peng, G. Li, Z. Yang, and J. Wu, "Classification of Peanut Images Based on Multi-features and SVM," IFACPapersOnLine, vol. 51, no. 17, pp. 726-731, 2018, doi: 10.1016/j.ifacol.2018.08.110.

[7] Z. Zhang, "Semi-supervised hyperspectral image classification algorithm based on graph embedding and discriminative spatial information," Microprocess. Microsyst., vol. 75, p. 103070, 2020, doi: 10.1016/j.micpro.2020.103070.

[8] J. Zhao, Q. Hu, G. Liu, X. Ma, F. Chen, and M. M. Hassan, "AFA: Adversarial fingerprinting authentication for deep neural networks," Comput. Commun., vol. 150, no. December 2019, pp. 488-497, 2020, doi: 10.1016/j.comcom.2019.12.016.

[9] I. G. Y. B. and A. Courville, "Deep Learning Ian," Foreign Aff., vol. 91, no. 5, pp. 1689-1699, 2012, doi: 10.1017/CBO9781107415324.004.

[10] M. Castelluccio, G. Poggi, C. Sansone, and L. Verdoliva, "Land Use Classification in Remote Sensing Images by Convolutional Neural Networks," no. September, 2015.

[11] Y. Chen, X. Zhao, and X. Jia, "Spectral-Spatial Classification of Hyperspectral Data Based on Deep Belief Network," IEEE J. Sel. Top. Appl. Earth Obs. Remote Sens., vol. 8, no. 6, pp. 2381-2392, 2015, doi: 10.1109/JSTARS.2015.2388577.

[12] "APPLICATION OF DEEP-LEARNING ALGORITHMS TO MSTAR DATA Haipeng Wang, Member, IEEE, Sizhe Chen , Student Member, IEEE, Feng Xu, Senior Member, IEEE and Ya-Qiu Jin, Fellow, IEEE Key Laboratory for Information Science of Electromagnetic Waves ( MoE )," IEEE Int. Geosci. Remote Sens. Symp. 2015, pp. 3743-3745, 2015.

[13] R. C. Hidayatullah and S. Violina, "Convolutional Neural Network Architecture and Data Augmentation for Pneumonia Classification from Chest X-Rays Images,” vol. 5, no. 2, pp. 158-164, 2020.

[14] W. S. Jeon and S. Y. Rhee, "Fingerprint pattern classification using convolution neural network," Int. J. Fuzzy Log. Intell. Syst., vol. 17, no. 3, pp. 170-176, 2017, doi: 10.5391/IJFIS.2017.17.3.170.

[15] Kaggle, "Sokoto Coventry Fingerprint Dataset (SOCOFing)." [Online]. Available: https://www.kaggle.com/ruizgara/socofing.

[16] L. Marifatul Azizah, S. Fadillah Umayah, and F. Fajar, "Deteksi Kecacatan Permukaan Buah Manggis Menggunakan Metode Deep Learning dengan Konvolusi Multilayer," Semesta Tek., vol. 21, no. 2, pp. 230-236, 2018, doi: 10.18196/st.212229.

[17] B. K. Rajan, N. Anto, and S. Jose, "Fusion of iris \& fingerprint biometrics for gender classification using neural network," $2 n d$ Int. Conf. Curr. Trends Eng. Technol. ICCTET 2014, pp. 216-221, 2014, doi: 10.1109/ICCTET.2014.6966290.

[18] R. S. Falasev, "Matriks Kookurensi Aras Keabuan ( Gray Level Co-Ocurrence Matrix )," no. August 2016, 2011.

[19] A. D. A. Veneza, "Fungsi Sidik Jari Dalam Mengidentifikasi Korban dan Pelaku Tindak Pidana," Universitas Hasanuddin, 2013.

[20] STIFIn, "Ilmu Rumusan Sidik Jari dan Tes Sidik Jari STIFIn." [Online]. Available: https://stifinfamily.com/ilmu-rumusansidik-jari-dan-tes-sidik-jari-stifin/. [Accessed: 15-Mar-2020].

[21] Investopedia, "Deep Learning." [Online]. Available: https://www.investopedia.com/terms/d/deep-learning.asp. [Accessed: 15Mar-2020]. 
[22] P. N. Rena, "Penerapan Metode Convolutional Neural Network Pada Pendeteksi Gambar Notasi Balok," Universitas Islam Negeri Syarif Hidayatullah Jakarta, 2019.

[23] K. He, X. Zhang, S. Ren, and J. Sun, "Deep residual learning for image recognition," Proc. IEEE Comput. Soc. Conf. Comput. Vis. Pattern Recognit., vol. 2016-Decem, pp. 770-778, 2016, doi: 10.1109/CVPR.2016.90.

[24] Y. Ju, X. Wang, and X. Chen, "Research on OMR recognition based on convolutional neural network tensorflow platform," Proc. - 2019 11th Int. Conf. Meas. Technol. Mechatronics Autom. ICMTMA 2019, pp. 688-691, 2019, doi: 10.1109/ICMTMA.2019.00157.

[25] S. R. DEWI, "Deep Learning Object Detection Pada Video Menggunakan Tensorflow Dan Convolutional Neural Network," Universitas Islam Indonesia, 2018.

[26] V. Rezende, M. Costa, A. Santos, and R. C. L. De Oliveira, "Image processing with convolutional neural networks for classification of plant diseases," Proc. - 2019 Brazilian Conf. Intell. Syst. BRACIS 2019, pp. 705-710, 2019, doi: 10.1109/BRACIS.2019.00128.

[27] P. Bashivan, I. Rish, M. Yeasin, and N. Codella, "Learning representations from EEG with deep recurrent-convolutional neural networks," 4th Int. Conf. Learn. Represent. ICLR 2016 - Conf. Track Proc., no. November, 2016.

[28] E. N. Arrofiqoh and H. Harintaka, "Implementasi Metode Convolutional Neural Network Untuk Klasifikasi Tanaman Pada Citra Resolusi Tinggi," Geomatika, vol. 24, no. 2, p. 61, 2018, doi: 10.24895/jig.2018.24-2.810.

[29] S. R. Putra, "Implementasi Convolutional Neural Network Untuk Klasifikasi Obyek Pada Citra," Institut Teknologi Sepuluh Nopember, 2015.

[30] U. Leiden and R. Magni, Deep Learning for Visual Understanding Proefschrift. 2017.

[31] K. P. Shung, "Accuracy, Precision, Recall or F1?" [Online]. Available: https://towardsdatascience.com/accuracy-precisionrecall-or-f1-331fb37c5cb9. [Accessed: 10-May-2020].

[32] C. Nicholson, "Evaluation Metrics for Machine Learning - Accuracy, Precision, Recall, and F1 Defined." [Online]. Available: https://pathmind.com/wiki/accuracy-precision-recall-f1. [Accessed: 10-May-2020].

\section{Authors' Profiles}

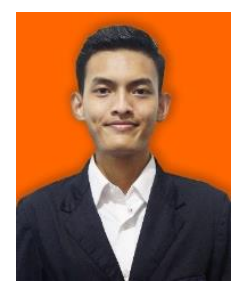

Ahmad Ilham Gustisyaf, born in Balikpapan, March 10, 1998. He lives at Bandung, West Java. He has attended elementary school at SDS Tamansiswa Jakarta, SMPN 14 Jakarta, and SMAN 1 Mimika.

Graduating from high school in 2016, he applied to study at Widyatama University, majoring in informatics. Now his concentration on his lectures, namely information and technology.

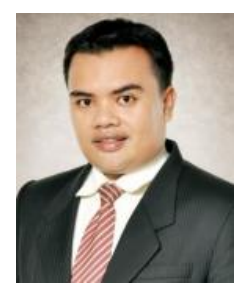

Ardiles Sinaga, is currently serving as a Lecturer on the Department of Informatics at Widyatama University and Binus University, Indonesia. In 2013, he is completed his S2-Magister in Telkom University on Informatics Department. He can be contacted at sinaga.diles@gmail.com.

How to cite this paper: Ahmad Ilham Gustisyaf, Ardiles Sinaga, " Implementation of Convolutional Neural Network to Classification Gender based on Fingerprint ", International Journal of Modern Education and Computer Science(IJMECS), Vol.13, No.4, pp. 55-67, 2021.DOI: 10.5815/ijmecs.2021.04.05 